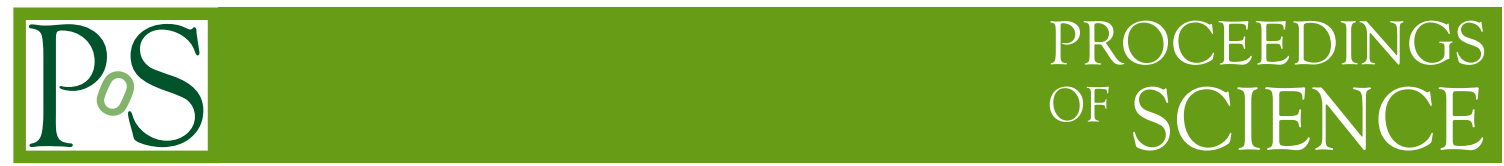

\title{
pA collisions in fixed-target mode at LHCb
}

\author{
Jihyun Bhom on behalf of the LHCb collaboration* \\ Instytut Fizyki Jadrowej Polskiej Akademii Nauk, Krakow, Poland \\ E-mail: jihyun.bhom@cern.ch
}

The LHCb experiment has the unique possibility to operate in the fixed target mode, using its internal gas target System for the Measuring the Overlap with Gas (SMOG). The energy scale achievable at the LHC and the excellent detector capabilities for vertexing, tracking and particle identification allow a wealth of measurements of great interest for cosmic ray and heavy ions physics. This talk presents recent results on antiproton production in the proton-helium collisions at $\sqrt{s_{N N}}=110 \mathrm{GeV}$ and the measurement of heavy-flavour production at the proton-helium collisions at $\sqrt{s_{N N}}=86.6 \mathrm{GeV}$.

XXXIX International Conference on High Energy Physics

3-11 July 2018

Seoul, South Korea

${ }^{*}$ Speaker. 


\section{Introduction}

The LHCb experiment [1] is a single-arm forward spectrometer covering the pseudorapidity range of $2<\eta<5$. Originally designed for precise luminosity measurements [2], the SMOG allows to inject noble gas such as Argon or Helium inside the primary LHC vacuum around the $\mathrm{LHCb}$ vertex detector (VELO). Since 2015, LHCb has started to exploit the SMOG to perform physics runs, using special fills not devoted to pp physics, with different beam and target configurations, allowing unique production studies which are relevant to cosmic ray and heavy ions physics [3].

\section{Results}

\section{$2.1 \bar{p}$ cross-section measurement}

The antiproton $(\bar{p})$ production crosssection in collisions of a $6.5 \mathrm{TeV}$ LHC proton beam on helium at rest is measured by the LHCb experiment using the SMOG internal gas target from a dataset corresponding to an integrated luminosity of $0.4 \mathrm{nb}^{-1}$. The $\bar{p}$ candidates are counted from a sample of 33.7 million reconstructed $\mathrm{pHe}$ collisions at $\sqrt{s_{N N}}=110 \mathrm{GeV}$. The results are illustrated in Figure 1. The double differential cross-section shape, notably the momentum spectrum, is found to be in a good agreement with the EPOS LHC simulation, while the absolute production rate is larger by about a factor 1.5 [4].

\subsection{Cross-sections of $J / \psi$ and $D^{0}$ mesons}

Production of prompt $J / \psi$ signal candidates at pHe collisions $\sqrt{s_{N N}}=86.6 \mathrm{GeV}$ is shown in Figure 2a. The mass fit functions are a sum of the Crystal Ball functions. The cross-section measurement of $J / \psi$ and $D^{0}$ as a function of the centre-of-mass energy $(\sqrt{s})$ are shown in Figure $2 \mathrm{~b}$ and Figure 2c. They are made for the pHe sample,

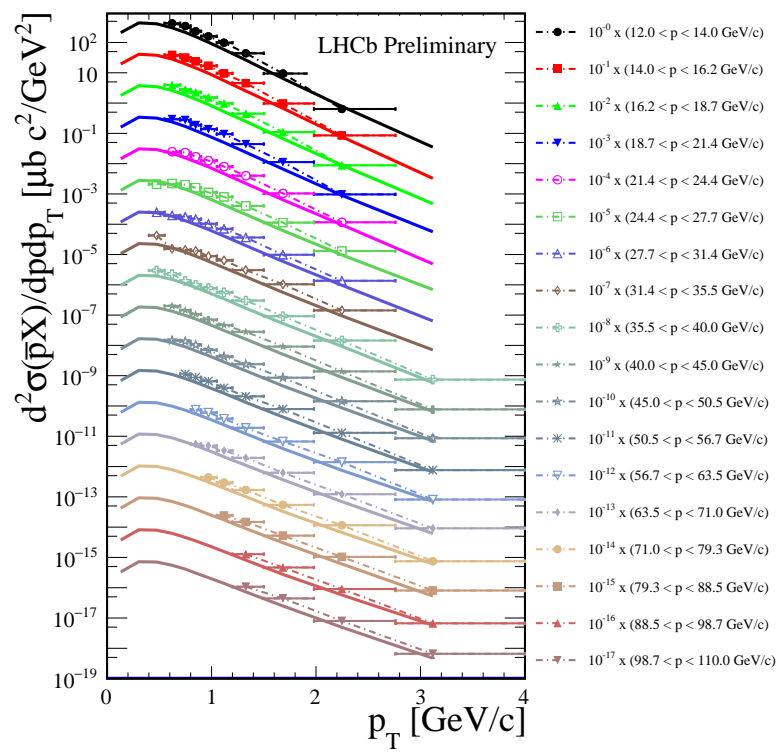

Figure 1: Result for the $\bar{p}$ cross-section measurement. The data points show the double differential cross-section as a function of $p_{T}$, with values successively scaled by a factor 0.1 to improve the readibility of the plot. The solid curves show the EPOS LHC absolute predictions, scaled with the same factors as the data. since only in this case the luminosity determination is available. The luminosity has been determined from a yield of the electrons elastically scattering off the He target [5]. With an integrated luminosity of $L_{p H e}=7.58 \pm 0.47 n b^{-1}$, the measured $J / \psi$ and $D^{0}$ cross-sections per target nucleon within $y \in[2,4.6]$, after correcting for the branching fractions of $J / \psi \rightarrow \mu^{+} \mu^{-}$and $D^{0} \rightarrow K^{-} \pi^{+}$, are measured to be 
$\sigma_{J / \psi}^{86.8 \mathrm{GeV}}=1225.6 \pm 62.0($ stat. $) \pm 81.6($ syst. $)$ nb/nucleon,

$\sigma_{D^{0}}^{86.8 \mathrm{GeV}}=156.0 \pm 4.6($ stat. $) \pm 12.3$ (syst.) $\mu \mathrm{b} /$ nucleon,

where first uncertainty is statistical (stat) and second is systematic (syst) and no systematic uncertainties have been assigned due to the extrapolation. In addition, the $D^{0}$ cross-section has been scaled with the global fragmentation factor, $f\left(c \rightarrow D^{0}\right)=0.542 \pm 0.024$, in order to obtain the $\bar{c} c$ production cross-section, $\sigma_{\bar{c} c}^{86.8 \mathrm{GeV}}=287.8 \pm 8.5$ (stat. $) \pm 25.7$ (syst.) $\mu \mathrm{b} /$ nucleon.

The $J / \psi$ differential cross-sections per target nucleon as a function of rapidity $\left(y^{*}\right)$ and transverse

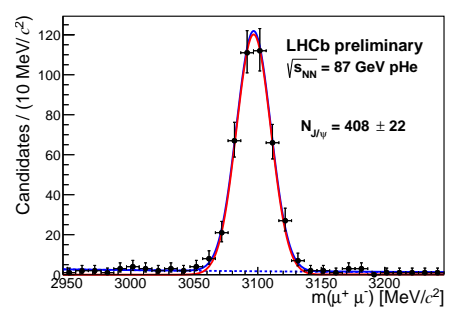

(a) $J / \psi \rightarrow \mu^{-} \mu^{+}$mass distributions with the fit functions.

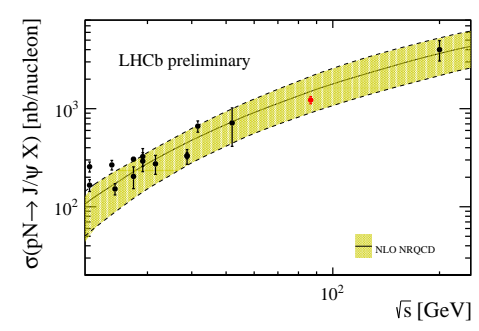

(b) $J / \psi$ cross-section measurements [6].

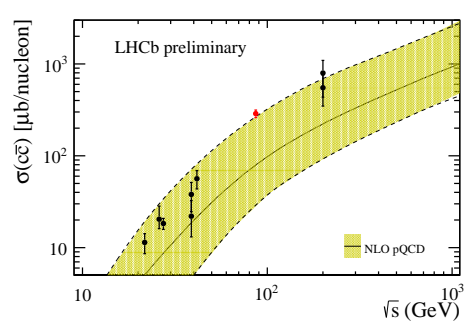

(c) $\bar{c} c$ cross-section measurements [7].

Figure 2: $J / \psi \rightarrow \mu^{-} \mu^{+}$mass distributions with the fit functions are shown (a). The black points are the data, the red line the signal, the dashed blue line the background, and the solid blue the sum of background and signal. Cross-section measurements of $J / \psi(b)$ and $\bar{c} c$ (c) are shown. The yellow band corresponds to NLO pQCD calculations [8]. Red points correspond to the pHe results.

momentum $\left(p_{T}\right)$ are shown in Figure $3 \mathrm{a}$ and Figure $3 \mathrm{~b}$. The $D^{0}$ differential cross-sections per target nucleon as a function of $y^{*}$ and $p_{T}$ are shown in Figure $3 \mathrm{c}$ and Figure $3 \mathrm{~d}$. The LHCb data are compared to phenomenological parameterizations, interpolated to the LHC energies.

\section{Summary}

The two measurements in the fixed target mode at the $\mathrm{LHCb}$ experiment have been presented. The $\bar{p}$ cross-section in collisions of a $6.5 \mathrm{TeV}$ LHC proton beam on helium at rest is measured from a dataset corresponding to an integrated luminosity of $0.4 n b^{-1}$. This is the first direct measurement of antimatter production in the $\mathrm{pHe}$ collisions and has an important implication for the measurement of an antiproton component in cosmic rays outside of the Earth's atmosphere [3]. The measurement of heavy flavor production in the fixed-target configuration at the LHC have been also measured. Those results imply that there is no evidence for any substantial intrinsic charm content of the nucleon.

\section{References}

[1] LHCb collaboration, The lhcb detector at the lhc, Journal of Instrumentation 3 (2008) S08005.

[2] LHCb collaboration, R. Aaij et al., Precision luminosity measurements at LHCb, JINST 9 (2014) P12005 [1410.0149]. 


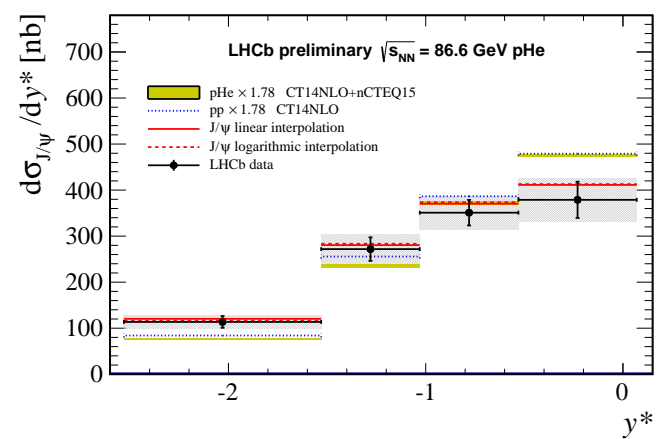

(a) $J / \psi$ cross section as a function of $y^{*}$.

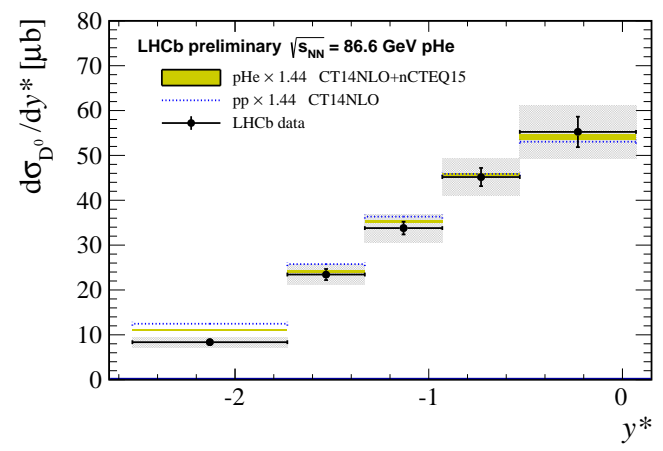

(c) $D^{0}$ cross section as a function of $y^{*}$.

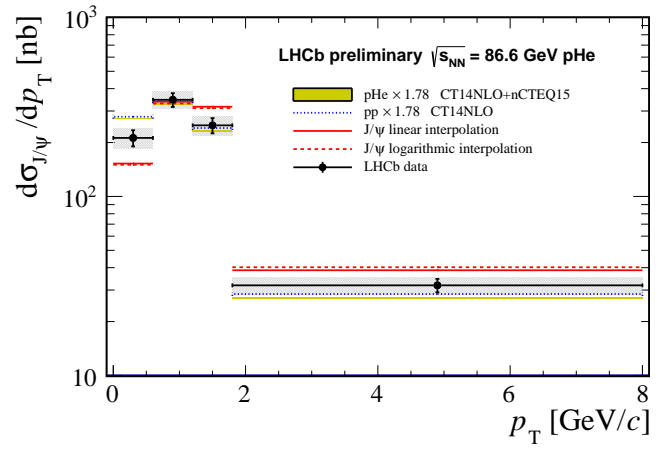

(b) $J / \psi$ cross section as a function of $p_{T}$.

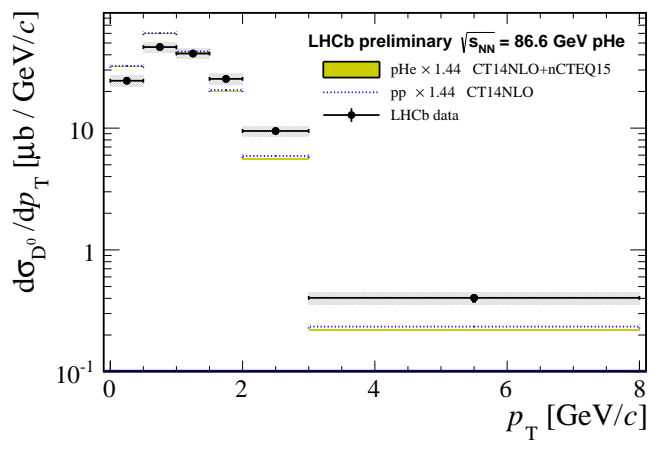

(d) $D^{0}$ cross section as a function of $p_{T}$.

Figure 3: $J / \psi$ cross section per nucleon as a function of $y^{*}$ (a) and $p_{T}$ (b) and $D^{0}$ cross section per nucleon as a function of $y^{*}(\mathrm{c})$ and $p_{T}$ (d) at $\mathrm{pHe} \sqrt{{s_{N N}}}=86.6 \mathrm{GeV}$, compared with theoretical predictions are shown. The uncertainty is the quadratic sum of the statistical and the systematic uncertainties.

[3] LHCb collaboration, E. Maurice, Fixed-target physics at LHCb, in 5th Large Hadron Collider Physics Conference (LHCP 2017) Shanghai, China, May 15-20, 2017, 2017, 1708.05184.

[4] LHCb collaboration, Measurement of antiproton production in pHe collisions at $\sqrt{s_{\mathrm{NN}}}=110 \mathrm{GeV}$, Tech. Rep. LHCb-CONF-2017-002. CERN-LHCb-CONF-2017-002, CERN, Geneva, Apr, 2017.

[5] LHCb collaboration, R. Aaij et al., Measurement of antiproton production in $\mathrm{pHe}$ collisions at $\sqrt{s_{N N}}=110 \mathrm{GeV}$, Submitted to: Phys. Rev. Lett. (2018) [1808.06127].

[6] F. Maltoni et al., Analysis of charmonium production at fixed-target experiments in the NRQCD approach, Phys. Lett. B638 (2006) 202 [hep-ph/ 0601203$].$

[7] ALICE collaboration, $d$ meson production in $p$ pb collisions at $\sqrt{s_{N N}}=5.02$ tev and in pp collisions at $\sqrt{s}=7$ tev, Phys. Rev. C 94 (2016) 054908.

[8] M. L. Mangano, P. Nason and G. Ridolfi, Heavy-quark correlations in hadron collisions at next-to-leading order, Nuclear Physics B 373 (1992) 295. 